\title{
EXPERIMENTAL INVESTIGATION AND SIMULATION OF 3D-PRINTED LATTICE STRUCTURES
}

\author{
Eva Heiml*, Anna Kalteis, Zoltan Major
}

\author{
Johannes Kepler Universität Linz, Institute of Polymer Product Engineering, Altenberger Straße 69, 4040 Linz, \\ Austria \\ * corresponding author: eva.heiml@jku.at
}

\begin{abstract}
Lattice structures are currently of high interest, especially for lightweight design. They generally have better structural performance per weight than parts made of bulk material. With conventional manufacturing techniques they are difficult to produce, but with additive manufacturing (AM) fabrication is feasible. To better understand their behaviour under various loading conditions two lattice structures in different configurations were observed. For each structure three different test specimens were designed and manufactured using selective laser sintering (SLS). To investigate the mechanical performance under large deformations the specimens were made of a thermoplastic polyurethane (TPU), which shows a hyperelastic material behaviour. Beside the experimental observations also finite element analyses (FEA) were conducted to investigate the deformation behaviour in more detail.
\end{abstract}

KEYWORDS: Lattice structures, additive manufacturing, mechanical testing, finite element analysis.

\section{INTRODUCTION}

Cellular structures are classified by their inner topology and are thus considered either as stochastic or as ordered. They can be further differentiated in open or closed cell types [1. Ordered materials generally have better mechanical properties, high surface area densities and lower pressure drops when compared to stochastic structures. The biggest disadvantage of stochastic cellular materials is the lack of design freedom. Therefore, ordered structures, especially lattice structures, are of higher interest [2]. Lattices have a trusslike structure with interconnected struts and nodes in a threedimensional space 3 . They consist of repeating unit cells, which have periodicity in three dimensions [1]. The differences in performance come from a different deformation behaviour. Foams are goverend by cell wall bending, whereas lattice cells stretch and compress [2]. This stretch dominated behaviour means that the initial yield is followed by either plastic buckling or brittle collapse which leads to post yield softening and then at the densification strain the stress rises steeply [4.

Ashby [4] has defined three design variables which describe the properties of cellular materials. The first variable is the material from which the structure is made. The second variable states that properties are different for varying cell topology and shape. And the third design variable is the relative density, which is the relation between the density of the cellular structure and that of the bulk material.

The aim of this work presented here is to model and investigate the behaviour of two lattice structures, manufactured with SLS, under tension and compression. The experimental data is compared to the results of the FEA. A further objective is to investigate this behaviour when the configuration of the specimen is changed.

\section{Test Specimen}

Two lattice structures are chosen to be investigated more thoroughly. One is the octet structure, Figure 1a. which consists of two regular tetrahedrons and one octahedron [5]. The second structure, Figure 1b, consists of a hexagonal array which forms the horizontal planes that are connected by spatial struts [6]. The models are created in NX 12.0 (Siemens PLM Software).

To create the specimens the diameter of the trusses as well as the length of each unit cell are adapted. Therefore, the overall dimension and the volume stay the same for each configuration. The overall dimensions were constrained by the building space of the SLS-printer, which only allowed $60 \mathrm{~mm}$ in each direction and a minimum diameter of $1.2 \mathrm{~mm}$. With three adjacent unit cells in each direction in space the basic lattice structure is created (Octet $3 \times 3 \times 3$, Hexagonal $3 \times 3 \times 3)$. To investigate the influence of increased number of unit cells another specimen with four cells is created (Octet $4 \times 4 \times 4$, Hexagonal $4 \times 4 \times 4)$. The third structure consists of five unit cells in two directions in space and three cells in the third one (Octet $5 \times 5 \times 3$, Hexagonal $5 \times 5 \times 3$ ) . To enable a uniform distribution of forces onto the specimen, $2.0 \mathrm{~mm}$ thick plates are added at the top and bottom of each structure. For the third specimen type these plates only cover an area of three times three unit cells, so that the forces apply on the same configuration as in the first specimen type. A summary of specimen data can be found in Table 1 For each specimen information about the dimension of the unit cell, including the diameter of the trusses $d$, the overall dimension and the area where the load is applied is given.

To make production easier and to reduce stress con- 


\begin{tabular}{lcccccccc}
\hline Specimen & \multicolumn{9}{c}{$\begin{array}{c}\text { Unit Cell Dimension } \\
{[\mathbf{m m}]}\end{array}$} & \multicolumn{3}{c}{$\begin{array}{c}\text { Cube Dimension } \\
{[\mathbf{m m}]}\end{array}$} & $\begin{array}{c}\text { Load Face Area } \\
{\left[\mathbf{m m}^{\mathbf{2}}\right]}\end{array}$ \\
& $\mathrm{x}$ & $\mathrm{y}$ & $\mathrm{z}$ & $\mathrm{d}$ & $\mathrm{x}$ & $\mathrm{y}$ & $\mathrm{z}$ & \\
\hline Octet $3 \times 3 \times 3$ & 20 & 20 & 20 & 3 & 60 & 60 & 60 & 3600 \\
Octet $4 \times 4 \times 4$ & 15 & 15 & 15 & 2.3 & 60 & 60 & 60 & 3600 \\
Octet $5 \times 5 \times 3$ & 12 & 12 & 12 & 1.8 & 60 & 60 & 36 & 1296 \\
Hexagonal $3 \times 3 \times 3$ & 11.5 & 11.5 & 19.91 & 3 & 34.5 & 34.5 & 59.75 & 1190.25 \\
Hexagonal $4 \times 4 \times 4$ & 8.6 & 8.6 & 14.89 & 2.3 & 34.4 & 34.4 & 59.58 & 1183.36 \\
Hexagonal $5 \times 5 \times 3$ & 6.9 & 6.9 & 11.95 & 1.8 & 34.5 & 34.5 & 35.83 & 428.49 \\
\hline
\end{tabular}

TABle 1. Specimen Data

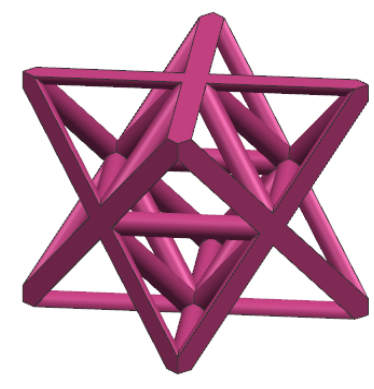

(A) . Octet Structure

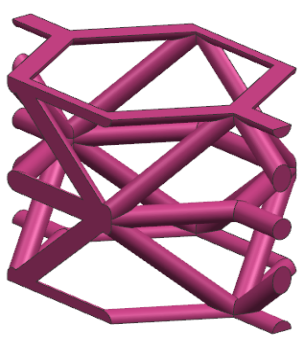

(B) . Hexagonal Structure

Figure 1. Lattice Structures

centrations radii are added at the strut connections. For the simulations they are removed, to enable a more regular mesh distribution. Test runs showed, that the influence of the radii on the outcome of the simulation is neglectable. The lattice structures have very complex geometries, thus, the chosen manufacturing method is SLS. This is a powder bed based process, where a laser beam selectively sinters material particles. This method is especially interesting for manufacturing lattice structures, because the unused powder can function as support material [7].

\section{Mechanical Testing}

\subsection{Bulk Material Characterisation}

Before the testing of the lattice structures started, a basic characterisation of the TPU was undertaken. For this, tension tests of ISO 527-5b specimens at different test rates were carried out. The results for $0.1 \mathrm{~mm} \cdot \mathrm{s}^{-1}$ were then used to create the material model. Results for the bulk material characterisation are depicted in Figure 2. These results show the time dependence and the hyperelastic material behaviour.

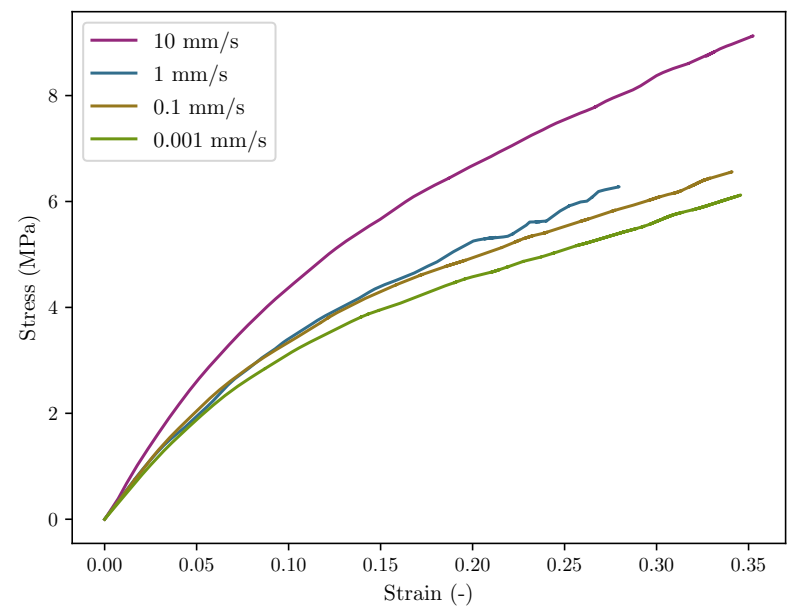

FiguRE 2. Bulk Material Characterisation

\subsection{Lattice Structure Characterisation}

All compression and tension testing was carried out on a MTS 852 test system at room temperature. The test speed of the machine was set to $0.1 \mathrm{~mm} \cdot \mathrm{s}^{-1}$. For compression tests the machine was set up in a way that the specimens rested on one compression plate while the other one applied the force from the top. For tension tests additional support structures, manufactured with fused deposition modelling (FDM) using acrylonitrile butadiene styrene (ABS), were glued on the specimens. The clamps were then mounted on those support structures (Figure 3). Tension tests were conducted until either failure of the bonding layer or failure of the lattice occured. Compression tests were stopped after reaching a certain strain. For strain evaluation digital image correlation (DIC) was used. 


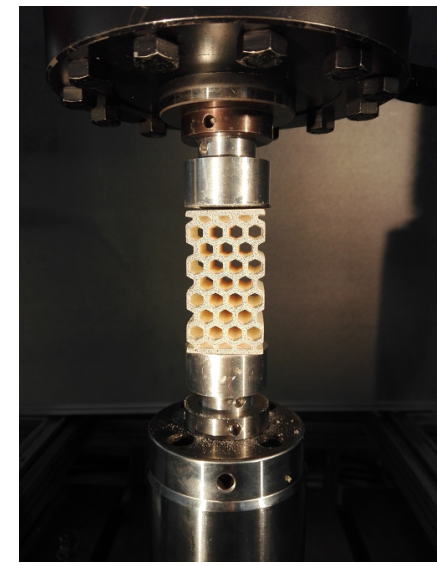

(A) . Compression Test

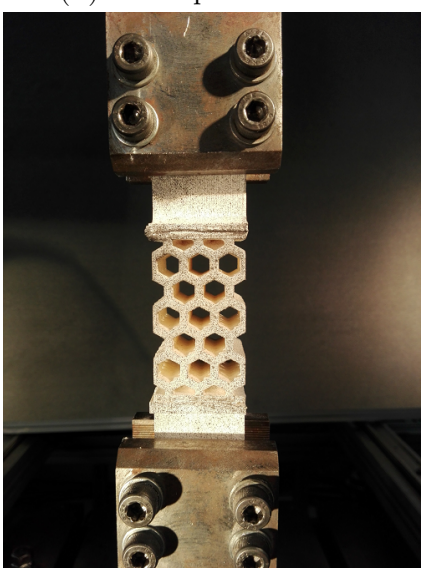

(в) . Tension Test

Figure 3. Test Setup

\section{Finite Element Analysis}

\subsection{Material Model Generation}

The data from the bulk material tests at $0.1 \mathrm{~mm} \cdot \mathrm{s}^{-1}$ is used to generate a hyperelastic material model for the TPU, because the experiments were performed with this test rate. The true strain results together with the true stress are then used to create the material model in Abaqus 6.14 (Dassault Systèmes). The Ogden model (see [8]) at a strain energy potential of order 3 best describes the actual behaviour of the TPU and is therefore used in the simulation, see Figure 4

\subsection{Simulation Methodology}

The FEA is performed using Abaqus as well. For compression tests rigid plates are added to the top and bottom of each structure. Through these the boundary conditions are applied. A frictionless contact between the plates and the specimen is created. The deformation is the same as in the mechanical testing in order to compare the results of the simulations and experiments. For tension tests reference points above and below the support structures, connected via coupling, and kinematic constraints are used to apply the boundary conditions (Figure 5). The support structures are included in the simulation, because they are made of ABS, which has a low stiffness as

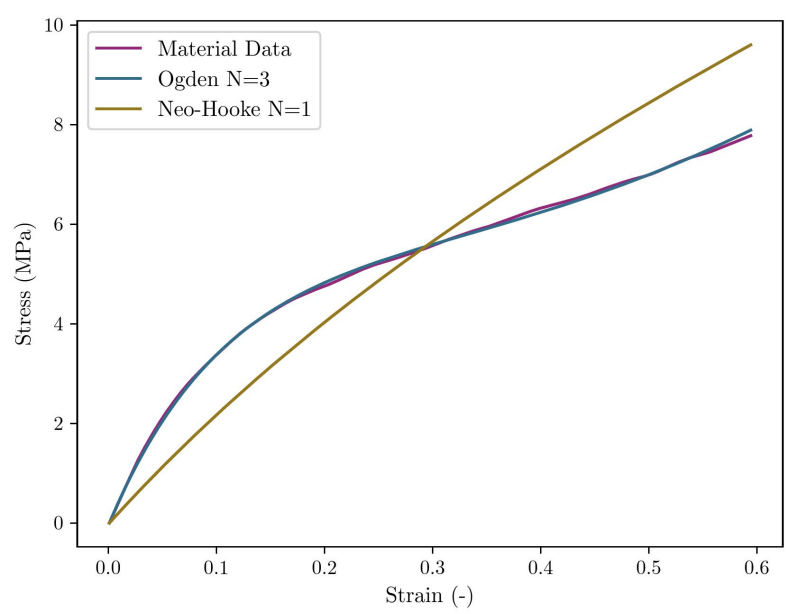

Figure 4. Material Model Generation

well. With linear tetrahedral elements a free mesh is created, on the bounding faces a mapped mesh is used. Seed sizes are adapted for each structure, with at least four elements over the thickness. The strain-rate sensitivity of the bulk material is identified (see Figure 2), this effect is neglected for material model used during FEA. Because of the time-independence of the material model it is possible to describe the test-rate of the experiments also with the explicit dynamic solver, which is used to improve computational efficiency, additionally mass scaling is utilised. Since the generated material model is time independent the quasi-static tests can be represented with an explicit solver. To simulate the behaviour of the TPU the hyperelastic material model from Figure 4 is implemented. Both simulations are displacement controlled.

\section{Results}

\subsection{EXPERIMENTS}

The apparent tensile modulus and apparent compressive modulus is calculated with Equation 1 in the standardised strain region $\left(\epsilon_{1}=0.05 \%, \epsilon_{2}=0.25 \%\right)$.

$$
E=\frac{\sigma_{2}-\sigma_{1}}{\epsilon_{2}-\epsilon_{1}}
$$

The apparent tensile stress is evaluated at $20 \%$ strain, since that is the highest strain where all structures can be compared. The apparent compressive plateau stress is evaluated for each structure as well (see Figure 6). These two values are normalized with the load face area. A summary of the results can be found in Table 2

The apparent tensile modulus increases for both structures when the number of unit cells is increased. The apparent compressive modulus shows the same behaviour for the octet structures. Both the apparent tensile stress and the apparent compressive stress increase for a higher number of unit cells. The Hexagonal $5 \times 5 \times 3$ specimen shows the highest values, except for the apparent compressive modulus. 


\begin{tabular}{lllll}
\hline Specimen & $\begin{array}{l}\text { Apparent Tensile } \\
\text { Modulus }\end{array}$ & $\begin{array}{l}\text { Apparent Tensile } \\
\text { Stress at 20 \% } \\
\text { Strain } \\
{[\mathrm{MPa}]}\end{array}$ & $\begin{array}{l}\text { Apparent } \\
\text { pressive } \\
\text { lus } \\
{[\mathrm{MPa}]}\end{array}$ & $\begin{array}{l}\text { Com- } \\
\text { Modu- }\end{array}$ \\
& {$[\mathrm{MPp}]$} & 0.4614 & 1.31 & $\begin{array}{l}\text { Aprent Com- } \\
\text { pressive Plateau } \\
{[\mathrm{MPa}]}\end{array}$ \\
\hline Octet $3 \times 3 \times 3$ & 3.61 & 0.5042 & 0.54 & 0.35 \\
Octet $4 \times 4 \times 4$ & 4.32 & 0.6640 & 0.11 & 0.43 \\
Octet $5 \times 5 \times 3$ & 5.47 & 0.7717 & 0.79 & 0.47 \\
Hexagonal $3 \times 3 \times 3$ & 6.74 & 0.8755 & 1.81 & 0.49 \\
Hexagonal $4 \times 4 \times 4$ & 7.53 & 1.5060 & 1.77 & 0.65 \\
Hexagonal $5 \times 5 \times 3$ & 15.51 & & & 1.76 \\
\hline
\end{tabular}

TABle 2. Experimental Results

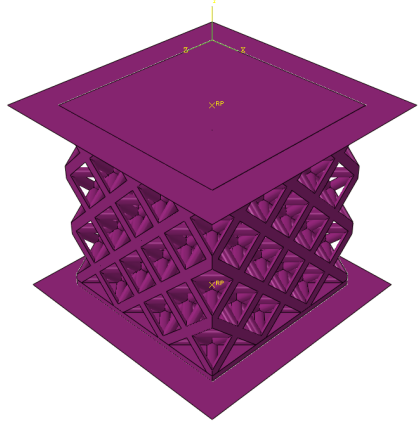

(A) . Compression Simulation

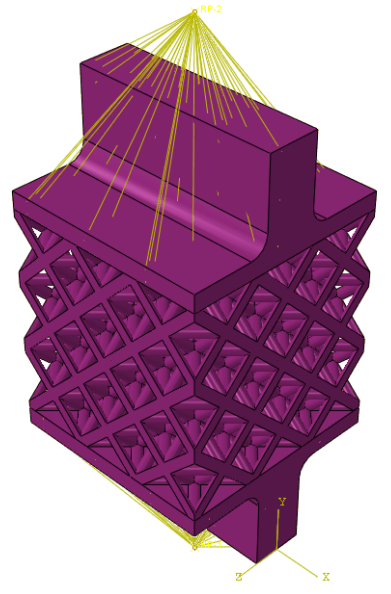

(в). Tension Simulation

Figure 5. Simulation Setup

\subsection{Simulation}

The experimental results are used to validate the simulations. The results of a range of selected structures are further discussed in this section. Figure 7 displays the behaviour of the Hexagonal $3 \times 3 \times 3$ and Hexagonal $4 \times 4 \times 4$ specimens under tension. For small strains both configurations show a linear elastic behaviour. For a higher number of unit cells the slope of the curves is higher, both for experiment and simulation. Especially for small displacements the results of experiment and simulation are in a good agreement.

The problem of most tension tests was, that the

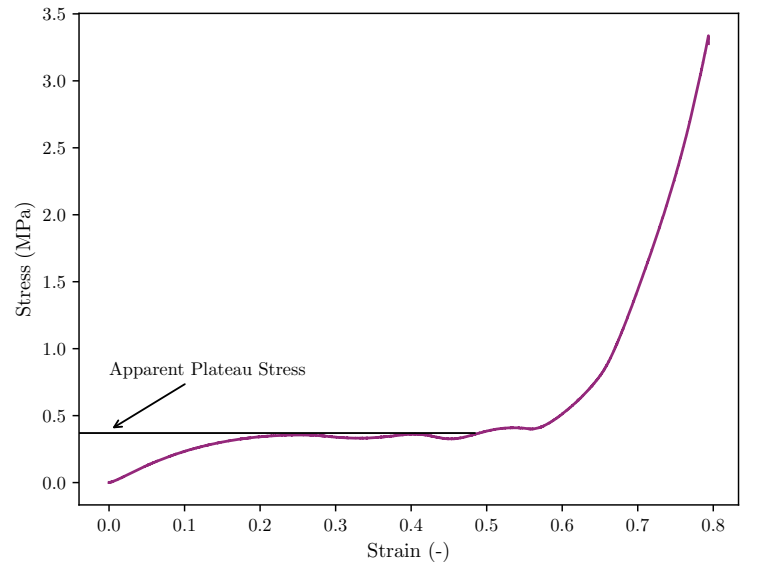

(A) . Compression Test

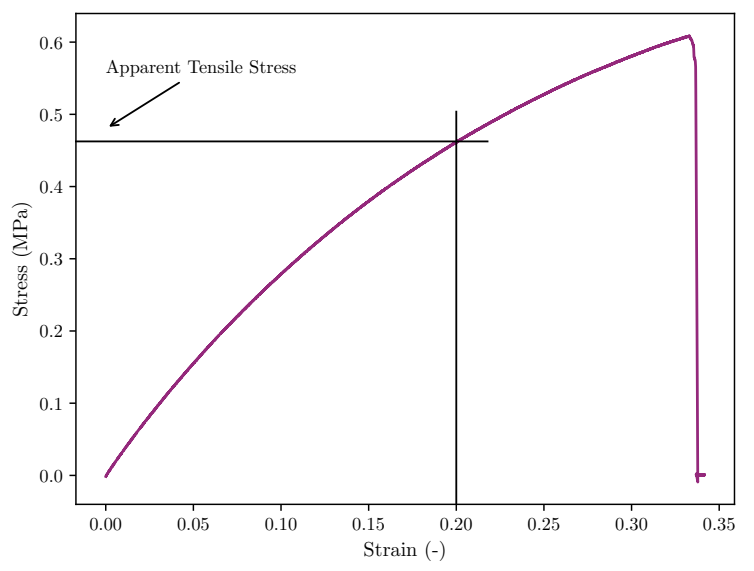

(в) . Tension Test

Figure 6. Evaluation of Test Results

bonding layer failed prior to the structure. Only for the specimens with five unit cells in $\mathrm{x}$ and $\mathrm{y}$ direction failure of the material occured. Figure 8 shows the results of the mechanical test and the simulation of the hexagonal structure shortly before the struts connecting the lattice structure with the support structure rip. Until this point the experimental result and the result of the simulation are in a good agreement.

Depicted in Figure 9 the struts start to rip at a displacement of around $7 \mathrm{~mm}$ and a force of $600 \mathrm{~N}$. This 


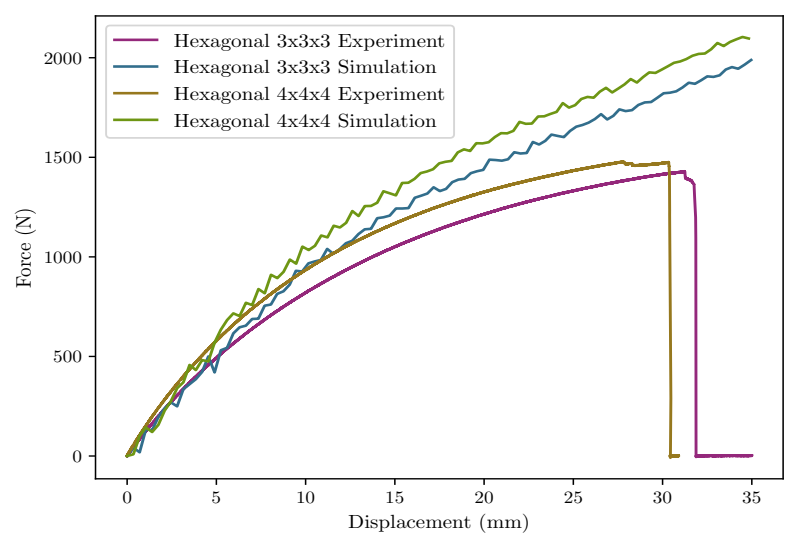

Figure 7. Tension Tests of Hexagonal $3 \times 3 \times 3$ and Hexagonal $4 \times 4 \times 4$
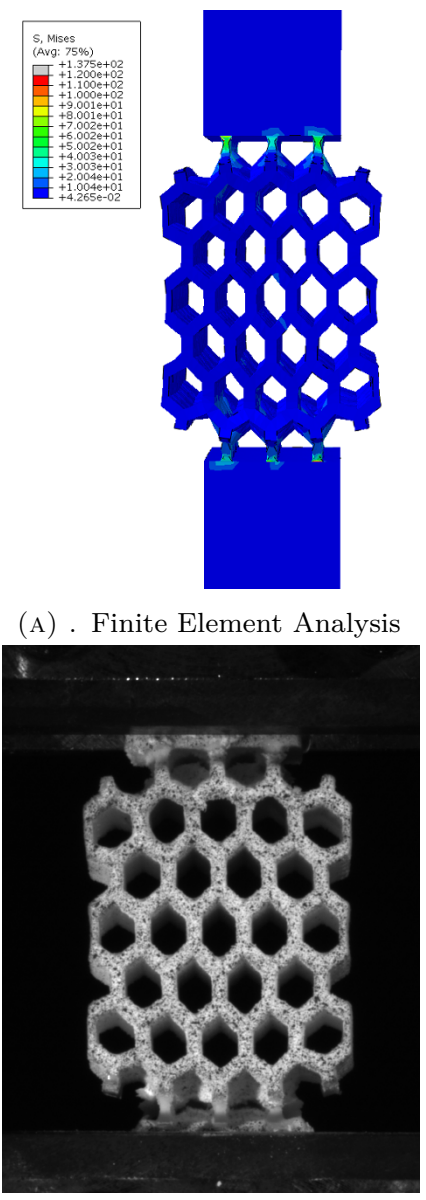

(в) . Mechanical Test

Figure 8 . Tension Test of Hexagonal $5 \times 5 \times 3$

is way less than what the other hexagonal structures endure, where the bonding layer failed at approximately $30 \mathrm{~mm}$ displacement and a force of $1400 \mathrm{~N}$. The force acts on the same number of unit cells as on the Hexagonal $3 \times 3 \times 3$ specimen, but on a smaller area. For the same number of unit cells their size is an important factor for how much load they can bear.

In Figure 10 the results for compression tests of the Octet $3 \times 3 \times 3$ and the Octet $4 \times 4 \times 4$ specimens are depicted. For small displacements the results of the

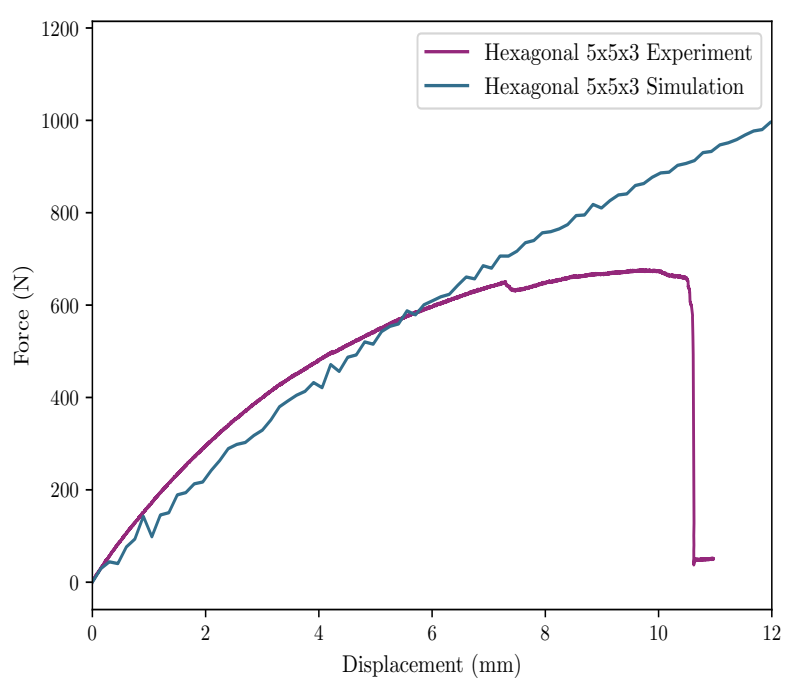

Figure 9. Tension Test of Hexagonal $5 \times 5 \times 3$

mechanical tests show a linear behaviour and match with the simulation for both specimens. Then again for a higher number of unit cells the force progression is higher. At a displacement of around $10 \mathrm{~mm}$ a force plateau is reached and at a later point the densification starts, which again happens earlier for Octet $4 \times 4 \times 4$.

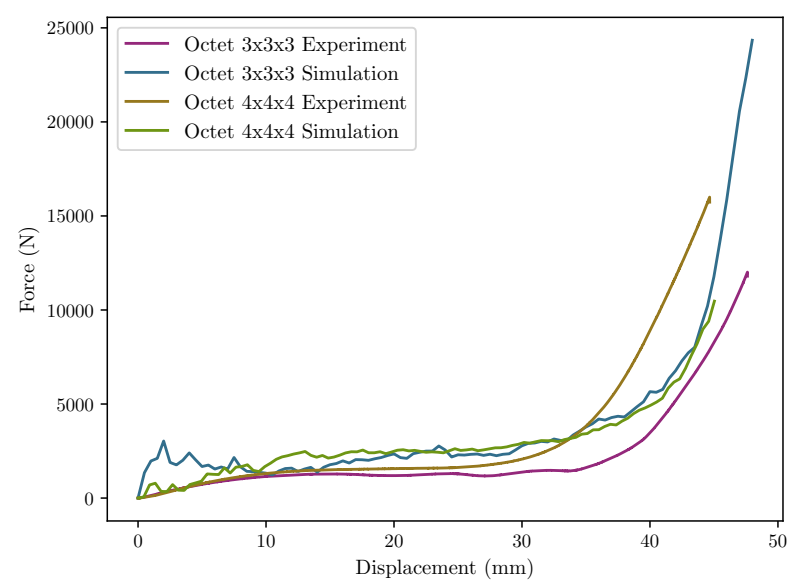

Figure 10. Compression Tests of Octet $3 \times 3 \times 3$ and Octet $4 \times 4 \times 4$

\section{Conclusion}

This paper investigates the behaviour of two different lattice structures with three different configurations under tension and compression. As it is described by Ashby 4 the geometry is a crucial factor for the behaviour of such structures. The specimens with three and four unit cells have the same volume for both structures respectively, thus the influence of the diameter on the apparent tensile modulus can be evaluated. As shown in Figure 11, the modulus increases when the diameter decreases.

It is assumed that stiffening is a result of internal constraint from adjacent unit cells, which increases 


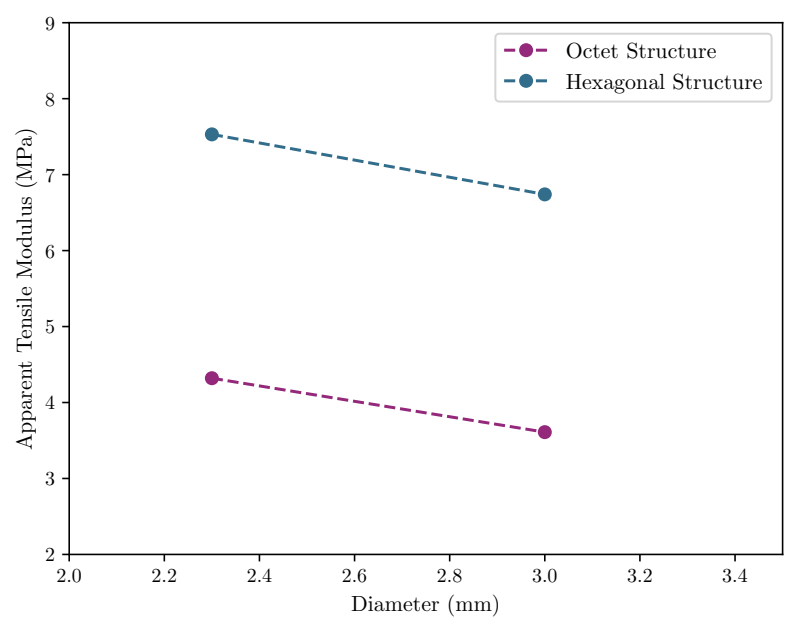

Figure 11. Influence of the Diameter on the Apparent Tensile Modulus

with the number of unit cells in the specimen. Specimens with smaller and thus more unit cells show a stiffer response. For the validation of this assumption further configurations have to be investigated. In a first step only a simple time-independent material model is implemented, which will be extended to consider the time-dependence of the material. The model created to describe the lattice structures, when tension and compression is applied, is in a good agreement with the experimental data. Since there is no failure criterion implemented, there are still some deviations, which can be resolved by expanding the model. With the method being validated by the experiments, it can be further developed for various loading conditions.

\section{ACKNOWLEDGEMENTS}

The specimens for the work conducted were manufactured at Bernstein Innovation $G m b H$. The mechanical tests were performed in the IPPE laboratory under the supervision of Michael Lackner.

\section{REFERENCES}

[1] T. George. Carbon fiber composite cellular structures, 2014. PhD Thesis, School of Engineering and Applied Science, University of Virginia.

[2] D. Rosen, S. R. Johnston, M. Reed. Design of general lattice structures for lightweight and compliance applications 2019 .

[3] G. Dong, Y. Tang, Y. Zhao. A survey of modeling of lattice structures fabricated by additive manufacturing. Journal of Mechanical Design 139, 2017. DOI:10.1115/1.4037305

[4] M. F. Ahsby. Cellular solids - scaling of properties. In Cellular Ceramics: Structure, Manufacturing, Properties and Applications, chap. 1.1. Wiley-VCH Verlag GmbH and Co. KGaA, Weinheim, 2005. DOI:10.1002/3527606696.ch1a

[5] R. Fuller. Synergetic building construction, 1961. US Patent 2,986,241.

[6] J. Aboudi, R. Gilat. Micromechanical analysis of lattice blocks. International Journal of Solids and Structures 42:4372-4392, 2005. DOI:10.1016/j.ijsolstr.2005.01.008

[7] W. Tao, M. Leu. Design of lattice structure for additive manufacturing. pp. 325-332. 2016. DOI:10.1109/ISFA.2016.7790182.

[8] R. Ogden. Large deformation isotropic elasticity: On the correlation of theory and experiments for incompressible rubber-like solids. Proc Roy Soc Lond Ser A: Math Phys Sci 326:556-585, 1992. DOI:10.1098/rspa.1972.0096 